\title{
Constraining the Parameters of Modified Chaplygin Gas in Einstein-Aether Gravity
}

\author{
Ujjal Debnath \\ Department of Mathematics, Indian Institute of Engineering Science and Technology, Shibpur, Howrah 711 103, India \\ Correspondence should be addressed to Ujjal Debnath; ujjaldebnath@yahoo.com
}

Received 19 September 2014; Revised 13 December 2014; Accepted 16 December 2014; Published 30 December 2014

Academic Editor: George Siopsis

Copyright ( 2014 Ujjal Debnath. This is an open access article distributed under the Creative Commons Attribution License, which permits unrestricted use, distribution, and reproduction in any medium, provided the original work is properly cited. The publication of this article was funded by SCOAP S $^{3}$

\begin{abstract}
We have assumed FRW model of the universe in Einstein-Aether gravity filled with dark matter and modified Chaplygin gas (MCG) type dark energy. We present the Hubble parameter in terms of some unknown parameters and observational parameters with the redshift $z$. From observed Hubble data (OHD) set (12 points), we have obtained the bounds of the arbitrary parameters $(A, B)$ of MCG by minimizing the $\chi^{2}$ test. Next due to joint analysis of BAO and CMB observations, we have also obtained the best fit values and the bounds of the parameters $(A, B)$ by fixing some other parameters. We have also taken type Ia supernovae data set (union 2 data set with 557 data points). Next due to joint analysis with $\mathrm{SNe}$, we have obtained the best fit values of parameters. The best fit values and bounds of the parameters are obtained by $66 \%, 90 \%$, and $99 \%$ confidence levels for OHD, OHD + BAO, OHD + BAO + $\mathrm{CMB}$, and $\mathrm{OHD}+\mathrm{BAO}+\mathrm{CMB}+\mathrm{SNe}$ joint analysis. The distance modulus $\mu(z)$ against redshift $z$ for our theoretical MCG model in Einstein-Aether gravity has been tested for the best fit values of the parameters and the observed SNe Ia union2 data sample.
\end{abstract}

\section{Introduction}

Observational evidence strongly points to an accelerated expansion of the universe, but the physical origin of this acceleration is unknown. The observations include type Ia supernovae and cosmic microwave background (CMB) [15] radiation. The standard explanation invokes an unknown "dark energy" component which has the property of positive energy density and negative pressure. Observations indicate that dark energy occupies about $70 \%$ of the total energy of the universe, and the contribution of dark matter is $~ 26 \%$. This accelerated expansion of the universe has also been strongly confirmed by some other independent experiments like Sloan Digital Sky Survey (SDSS) [6], baryonic acoustic oscillation (BAO) [7], WMAP data analysis $[8,9]$, and so forth. Over the past decade there have been many theoretical models for mimicking the dark energy behaviors, such as the simplest (just) cosmological constant in which the equation of state is independent of the cosmic time and which can fit the observations well. This model is the so-called $\Lambda \mathrm{CDM}$, containing a mixture of cosmological constant $\Lambda$ and cold dark matter (CDM). However, two problems arise from this scenario, namely "fine-tuning" and the "cosmic coincidence" problems. In order to solve these two problems, many dynamical dark energy models were suggested, whose equation of state evolves with cosmic time. The scalar field or quintessence $[10,11]$ is one of the most favored candidates of dark energy which produce sufficient negative pressure to drive acceleration. In order to alleviate the cosmologicalconstant problems and explain the acceleration expansion, many dynamical dark energy models have been proposed, such as K-essence, tachyon, phantom, quintom, and Chaplygin gas model [12-16]. Also the interacting dark energy models including modified Chaplygin gas [17], holographic dark energy model [18], and braneworld model [19] have been proposed. Recently, based on principle of quantum gravity, the agegraphic dark energy (ADE) and the new agegraphic dark energy (NADE) models were proposed by Cai [20] and Wei and Cai [21], respectively. The theoretical models have been tally with the observations with different data sets say TORNY, Gold sample data sets [3, 22-24]. In Einstein's gravity, the modified Chaplygin gas [17] best fits with the 3year WMAP and the SDSS data with the choice of parameters 
$A=0.085$ and $\alpha=1.724$ [25] which are improved constraints compared to the previous ones $-0.35<A<0.025$ [26].

Another possibility is that general relativity is only accurate on small scales and has to be modified on cosmological distances. One of these is modified gravity theories. In this case cosmic acceleration would arise not from dark energy as a substance but rather from the dynamics of modified gravity. Modified gravity constitutes an interesting dynamical alternative to $\Lambda \mathrm{CDM}$ cosmology in that it is also able to describe the current acceleration in the expansion of our universe. The simplest modified gravity is DGP brane-world model [27]. The other alternative approach dealing with the acceleration problem of the Universe is changing the gravity law through the modification of action of gravity by means of using $f(R)$ gravity $[28,29]$ instead of the Einstein-Hilbert action. Some of these models, such as $1 / R$ and logarithmic models, provide an acceleration for the universe at the present time [30]. Other modified gravity includes $f(T)$ gravity, $f(G)$ gravity, Gauss-Bonnet gravity, Horava-Lifshitz gravity, and Brans-Dicke gravity [31-35].

In the present work, we concentrate on the generalized Einstein-Aether theories as proposed by Zlosnik et al. [36, 37], which is a generalization of the Einstein-Aether theory developed by Jacobson and Mattingly [38, 39]. These years a lot of work has been done in generalized Einstein-aether theories [40-46]. In the generalized Einstein-Aether theories by taking a special form of the Lagrangian density of Aether field, the possibility of Einstein-Aether theory as an alternative to dark energy model is discussed in detail, that is, taking a special Aether field as a dark energy candidate and the constraints have been found from observational data $[47,48]$. Since modified gravity theory may be treated as alternative to dark energy, so Meng and Du [47, 48] have not taken by hand any types of dark energy in EinsteinAether gravity and shown that the gravity may generate dark energy. Here if we exempt this assumption, so we need to consider the dark energy from outside. So we assume the FRW universe in Einstein-Aether gravity model filled with the dark matter and the modified Chaplygin gas (MCG) type dark energy. The basic concepts of Einstein-Aether gravity theory are presented in Section 2. The modified Friedmann equations and their solutions are given in Section 3. The observational data analysis tools in observed Hubble data $(\mathrm{OHD}), \mathrm{OHD}+\mathrm{BAO}, \mathrm{OHD}+\mathrm{BAO}+\mathrm{CMB}$, and $\mathrm{OHD}+\mathrm{BAO}$ $+\mathrm{CMB}+\mathrm{SNe}$ for $\chi^{2}$ minimum test will be studied in Section 4 and investigate the bounds of unknown parameters $(A, B)$ of MCG dark energy by fixing other parameters. The best fit values of the parameters are obtained by $66 \%, 90 \%$, and $99 \%$ confidence levels. The distance modulus $\mu(z)$ against redshift $z$ for our theoretical model of the MCG in Einstein-Aether gravity models for the best fit values of the parameters and the observed SNe Ia union2 data sample. Finally we present the conclusions of the work in Section 5.

\section{Einstein-Aether Gravity Theory}

In order to include Lorentz symmetry violating terms in gravitation theories, apart from some noncommutative gravity models, one may consider existence of preferred frames. This can be achieved admitting a unit timelike vector field in addition to the metric tensor of spacetime. Such a timelike vector implies a preferred direction at each point of spacetime. Here the unit timelike vector field is called the Aether and the theory coupling the metric and unit timelike vector is called the Einstein-Aether theory [38]. So Einstein-Aether theory is the extension of general relativity (GR) that incorporates a dynamical unit timelike vector field (i.e., Aether). In the last decade there is an increasing interest in the Aether theory.

The action of the Einstein-Aether gravity theory with the normal Einstein-Hilbert part action can be written in the form $[36,47]$

$$
S=\int d^{4} x \sqrt{-g}\left[\frac{R}{16 \pi G}+\mathscr{L}_{E A}+\mathscr{L}\right]
$$

where $\mathscr{L}_{E A}$ is the vector field Lagrangian density while $\mathscr{L}$ denotes the Lagrangian density for all other matter fields. The Lagrangian density for the vector part consists of terms quadratic in the field $[36,47]$ :

$$
\begin{gathered}
\mathscr{L}_{E A}=\frac{M^{2}}{16 \pi G} F(K)+\frac{1}{16 \pi G} \lambda\left(A^{a} A_{a}+1\right), \\
K=M^{-2} K_{c d}^{a b} \nabla_{a} A^{c} \nabla_{b} A^{d}, \\
K_{c d}^{a b}=c_{1} g^{a b} g_{c d}+c_{2} \delta_{c}^{a} \delta_{d}^{b}+c_{3} \delta_{d}^{a} \delta_{c}^{b},
\end{gathered}
$$

where $c_{i}$ are dimensionless constants, $M$ is the coupling constant which has the dimension of mass, $\lambda$ is a Lagrange multiplier that enforces the unit constraint for the time-like vector field, $A^{a}$ is a contravariant vector, $g_{a b}$ is metric tensor, and $F(K)$ ia an arbitrary function of $K$. From (1), we get the field equations

$$
\begin{gathered}
G_{a b}=T_{a b}^{E A}+8 \pi G T_{a b} \\
\nabla_{a}\left(F^{\prime} J_{b}^{a}\right)=2 \lambda A_{b}
\end{gathered}
$$

where

$$
F^{\prime}=\frac{d F}{d K}, \quad J_{b}^{a}=2 K_{b c}^{a d} \nabla_{d} A^{c} .
$$

Here $T_{a b}$ is the energy momentum tensor for all matter fluids and $T_{a b}^{E A}$ is the energy momentum tensor for the vector field and they are, respectively, given as follows: [47]

$$
T_{a b}=(\rho+p) u_{a} u_{b}+p g_{a b},
$$

where $\rho$ and $p$ are, respectively, the energy density and pressure of all matter fluids and $u_{a}=(1,0,0,0)$ is the fluid 4 -velocity vector and

$$
\begin{aligned}
T_{a b}^{E A}= & \frac{1}{2} \nabla_{d}\left[\left(J_{(a}{ }^{d} A_{b)}-J_{(a}^{d} A_{b)}-J_{(a b)} A^{d}\right) F^{\prime}\right] \\
& -Y_{(a b)} F^{\prime}+\frac{1}{2} g_{a b} M^{2} F+\lambda A_{a} A_{b}
\end{aligned}
$$

with

$$
Y_{a b}=-c_{1}\left[\left(\nabla_{d} A_{a}\right)\left(\nabla^{d} A_{b}\right)-\left(\nabla_{a} A_{d}\right)\left(\nabla_{b} A^{d}\right)\right],
$$

where the subscript $(a b)$ means symmetric with respect to the indices involved and $A^{a}=(1,0,0,0)$ is nonvanishing timelike unit vector satisfying $A^{a} A_{a}=-1$. 


\section{Modified Friedmann Equations and Solutions}

We consider the Friedmann-Robertson-Walker (FRW) metric of the universe as

$$
d s^{2}=-d t^{2}+a^{2}(t)\left[\frac{d r^{2}}{1-k r^{2}}+r^{2}\left(d \theta^{2}+\sin ^{2} \theta d \phi^{2}\right)\right],
$$

where $k(=0, \pm 1)$ is the curvature scalar and $a(t)$ is the scale factor. From (3) and (4), we get

$$
K=M^{-2}\left(c_{1} g^{a b} g_{c d}+c_{2} \delta_{c}^{a} \delta_{d}^{b}+c_{3} \delta_{d}^{a} \delta_{c}^{b}\right)=\frac{3 \beta H^{2}}{M^{2}},
$$

where $\beta=c_{1}+3 c_{2}+c_{3}$ is constant. From (5), we get the modified Friedmann equation for Einstein-Aether gravity as follows $[36,47]$ :

$$
\begin{aligned}
& \beta\left(-F^{\prime}+\frac{F}{2 K}\right) H^{2}+\left(H^{2}+\frac{k}{a^{2}}\right)=\frac{8 \pi G}{3} \rho, \\
& \beta \frac{d}{d t}\left(H F^{\prime}\right)+\left(-2 \dot{H}+\frac{2 k}{a^{2}}\right)=8 \pi G(\rho+p),
\end{aligned}
$$

where $H(=\dot{a} / a)$ is Hubble parameter. Now we see that if the first expressions of L.H.S. of (12) and (13) are zero, we get the usual field equations for Einstein's gravity. So first expressions arise for Einstein-Aether gravity. Also the conservation equation is given by

$$
\dot{\rho}+3 \frac{\dot{a}}{a}(\rho+p)=0
$$

Now, assume that the matter fluid is combination of dark matter and modified Chaplygin gas type dark energy. So $\rho=\rho_{m}+\rho_{c h}$ and $p=p_{m}+p_{c h}$, where $\rho_{m}$ and $p_{m}$ are, respectively, the energy density and pressure of dark matter and $\rho_{c h}$ and $p_{c h}$ are, respectively, the energy density and pressure of modified Chaplygin gas. Assume that the dark matter follows the barotropic equation of state $p_{m}=w_{m} \rho_{m}$, where $w_{m}$ is a constant. The equation of state of modified Chaplygin gas (MCG) is given by [17]

$$
p_{c h}=A \rho_{c h}-\frac{B}{\rho_{c h}^{\alpha}},
$$

where $A>0, B>0$, and $0 \leq \alpha \leq 1$. Now we assume that there is no interaction between dark matter and dark energy. So they are separately conserved. From (14), we obtain the conservation equations for dark matter and dark energy in the form:

$$
\dot{\rho}_{m}+3 \frac{\dot{a}}{a}\left(\rho_{m}+p_{m}\right)=0, \quad \dot{\rho}_{c h}+3 \frac{\dot{a}}{a}\left(\rho_{c h}+p_{c h}\right)=0 .
$$

Using equation of states and the conservation equations (17), we obtain $\rho_{m}=\rho_{m 0}(1+z)^{3\left(1+w_{m}\right)}$ and

$$
\rho_{c h}=\left[\frac{B}{1+A}+C(1+z)^{3(1+A)(1+\alpha)}\right]^{1 /(1+\alpha)},
$$

where $\rho_{m 0}$ and $C$ are positive constants in which $\rho_{m 0}$ represents the present value of the density of dark matter and $z=1 / a-1$ is the cosmological redshift (choosing $a_{0}=1$ ). The above expression can be written in the form:

$$
\rho_{c h}=\rho_{c h 0}\left[A_{s}+\left(1-A_{s}\right)(1+z)^{3(1+A)(1+\alpha)}\right]^{1 /(1+\alpha)},
$$

where $\rho_{c h 0}$ is the present value of the MCG density and $A_{s}=$ $B /((1+A) C+B)$. So $0 \leq A_{s} \leq 1$.

Now since $F(K)$ is a free function of $K$. Some authors have chosen $F(K)$ in the following forms: (i) $F(K)=\gamma(-K)^{n}$ [36, 43], (ii) $F(K)=\gamma \sqrt{-K}+\sqrt{3 K / \beta} \ln (-K)[47,48]$. Here we may choose another form of $F(K)$ for our next calculations in simplified form as $F(K)=(2 / \beta) K(1-\epsilon K)$, where $\epsilon$ is a constant. So solving (13), we obtain the expression of $H^{2}$ in terms of redshift $z$ in the following:

$$
\begin{aligned}
& H^{2}(z) \\
& =\frac{M}{3 \sqrt{3 \epsilon \beta}} \\
& \times\left[-3 k(1+z)^{2}+8 \pi G \rho_{m 0}(1+z)^{3\left(1+w_{m}\right)}\right. \\
& \left.\quad+8 \pi G \rho_{c h 0}\left\{A_{s}+\left(1-A_{s}\right)(1+z)^{3(1+A)(1+\alpha)}\right\}^{1 /(1+\alpha)}\right]^{1 / 2}
\end{aligned}
$$

Now defining the dimensionless parameters $\Omega_{m 0}=$ $8 \pi G \rho_{m 0} / 3 H_{0}^{2}, \Omega_{c h 0}=8 \pi G \rho_{c h 0} / 3 H_{0}^{2}, \Omega_{k 0}=k / H_{0}^{2}$, and $\Omega_{E A}=$ $M / 3 H_{0} \sqrt{\epsilon \beta}$, we obtain the form of $H(z)$ :

$H(z)$

$$
\begin{aligned}
= & H_{0} \sqrt{\Omega_{E A}} \\
\times & {\left[-\Omega_{k 0}(1+z)^{2}+\Omega_{m 0}(1+z)^{3\left(1+w_{m}\right)}\right.} \\
& \left.+\Omega_{c h 0}\left\{A_{s}+\left(1-A_{s}\right)(1+z)^{3(1+A)(1+\alpha)}\right\}^{1 /(1+\alpha)}\right]^{1 / 4} .
\end{aligned}
$$

Due to the above solution, (13) gives the following relation:

$$
\sqrt{\Omega_{E A}}\left[\Omega_{m 0}+\Omega_{c h 0}-\Omega_{k 0}\right]=1 .
$$

\section{Observational Data Analysis Tools}

In this section, we will investigate some bounds of the parameters of the modified Chaplygin gas (MCG) in EinsteinAether gravity by observational data fitting. The parameters are determined by observed Hubble data (OHD), BAO, CMB, and $\mathrm{SNe}$ data analysis [47-57]. We will use the $\chi^{2}$ minimization technique (statistical data analysis) from Hubble-redshift data set to get the constraints of the parameters of MCG model in Einstein-Aether gravity. 
4.1. Analysis with Observed Hubble Data (OHD). We analyze the MCG model in Einstein-Aether gravity using observed value of Hubble parameter data (OHD) $[58,59]$ at different redshifts consisting of twelve data points. The observed values of Hubble parameter $H(z)$ and the standard error $\sigma(z)$ for different values of redshift $z$ are listed in Table 1 . The $\chi^{2}$ statistics for $\mathrm{OHD}$ is given as follows:

$$
\chi_{\mathrm{OHD}}^{2}=\sum \frac{\left(H(z)-H_{\mathrm{obs}}(z)\right)^{2}}{\sigma^{2}(z)}
$$

where $H(z)$ and $H_{\text {obs }}(z)$ are, respectively, the theoretical and observational values of Hubble parameter at different redshifts and $\sigma(z)$ is the corresponding error which is given in Table 1. We consider the present value of Hubble parameter $H_{0}=72 \pm 8 \mathrm{Kms}^{-1} \mathrm{Mpc}^{-1}$. Here we will determine two parameters of MCG model out of 3 parameters $A, B, \alpha$ by fixing any one parameter from minimizing the above distribution $\chi_{\mathrm{OHD}}^{2}$. There are other parameters of the model say $\Omega_{m 0}, \Omega_{k 0}, \Omega_{c h 0}, \Omega_{E A}, w_{m}$. Fixing the one parameter $\alpha$ of MCG model, the relation between the other parameters $(A, B)$ can be determined by the observational data. Now for OHD analysis, $\chi_{\mathrm{OHD}}^{2}$ is minimized for best fit values of $A=0.238303$ and $B=0.18176$ and the minimum value of $\chi_{\text {OHD }}^{2}=7.08613$ where we have assumed $\alpha=0.1$. We also plot the graph for different confidence levels $(66 \%, 90 \%, 99 \%)$ in Figure 1.

4.2. Analysis with $\mathrm{OHD}+\mathrm{BAO}$. Another constraint is from the baryonic acoustic oscillations (BAO) traced by the Sloan Digital Sky Survey (SDSS). The BAO peak parameter value has been proposed by Eisenstein et al. [7]. Here we examine the parameters $A$ and $B$ for MCG gas model from the measurements of the $\mathrm{BAO}$ peak for low redshift (with range $0<z<0.35$ ) using standard $\chi^{2}$ analysis. The BAO peak parameter may be defined by [47]

$$
\mathscr{A}=\frac{\sqrt{\Omega_{m}}}{\left\{\Omega_{k} E\left(z_{1}\right)\right\}^{1 / 3}}\left[\frac{1}{z_{1}} \sinh \left\{\sqrt{\Omega_{k}} \int_{0}^{z_{1}} \frac{d z}{E(z)}\right\}\right]^{2 / 3},
$$

where $E(z)=H(z) / H_{0}$ may be called the normalized Hubble parameter and the redshift $z_{1}=0.35$ is the typical redshift of the SDSS. The value of the parameter $\mathscr{A}$ for the universe is given by $\mathscr{A}=0.469 \pm 0.017$ using SDSS data [7]. Now the $\chi^{2}$ function for the BAO measurement can be written as

$$
\chi_{\mathrm{BAO}}^{2}=\frac{(\mathscr{A}-0.469)^{2}}{(0.017)^{2}} .
$$

Now the total joint data analysis of BAO with OHD for the $\chi^{2}$ function may be defined by

$$
\chi_{\text {total }}^{2}=\chi_{\mathrm{OHD}}^{2}+\chi_{\mathrm{BAO}}^{2} .
$$

According to $\mathrm{OHD}+\mathrm{BAO}$ joint analysis the best fit values of $A$ and $B$ are $A=0.238695$ and $B=0.209932$ with $\chi^{2}$ minimum being 7.07842. Finally we draw the contours $B$ versus $A$ for the $66 \%, 90 \%$, and $99 \%$ confidence limits depicted in Figure 2.
TABLE 1: The observed Hubble parameter $H(z)$ and the standard error $\sigma(z)$ for different values of redshift $z$.

\begin{tabular}{lcc}
\hline$z$ & $H(z)$ & $\sigma(z)$ \\
\hline 0 & 73 & \pm 8 \\
0.1 & 69 & \pm 12 \\
0.17 & 83 & \pm 8 \\
0.27 & 77 & \pm 14 \\
0.4 & 95 & \pm 17.4 \\
0.48 & 90 & \pm 60 \\
0.88 & 97 & \pm 40.4 \\
0.9 & 117 & \pm 23 \\
1.3 & 168 & \pm 17.4 \\
1.43 & 177 & \pm 18.2 \\
1.53 & 140 & \pm 14 \\
1.75 & 202 & \pm 40.4 \\
\hline
\end{tabular}

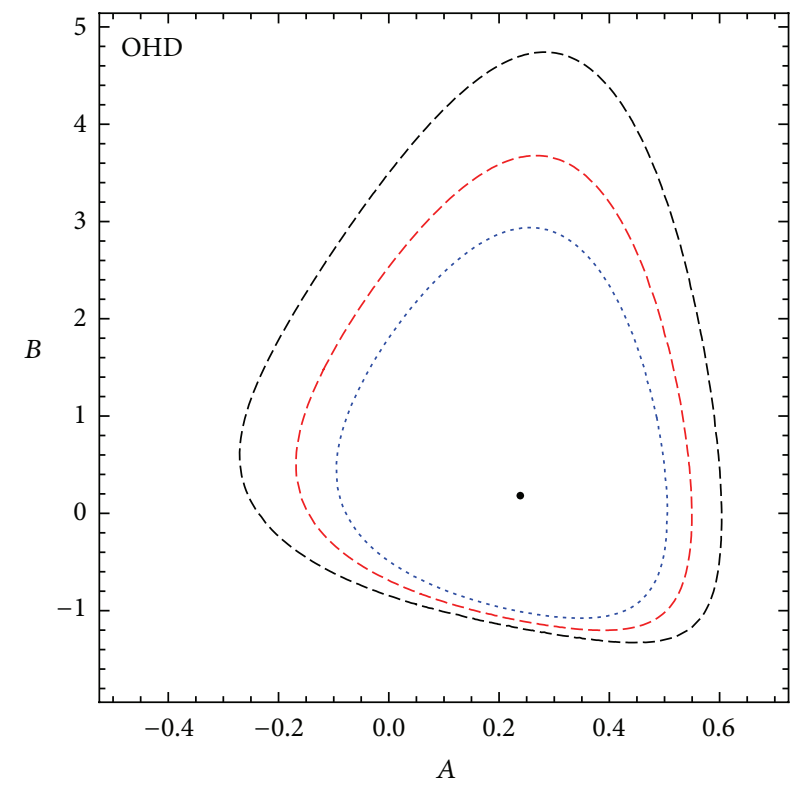

FIGURE 1: It shows the variations of $A$ with $B$ for $\alpha=0.1$ for OHD and $\mathrm{OHD}+\mathrm{BAO}$ analysis, respectively, for different confidence levels say $66 \%$ (solid, blue), 90\% (dashed, red), and 99\% (dashed, black) contours.

4.3. Analysis with $\mathrm{OHD}+B A O+C M B$. In addition to $\mathrm{OHD}$ and $\mathrm{BAO}$ analysis, we use the cosmic microwave background (CMB) shift parameter. The $\mathrm{CMB}$ shift parameter (CMB power spectrum first peak) is defined by [60-62]

$$
\mathscr{R}=\frac{\sqrt{\Omega_{m}}}{\sqrt{\Omega_{k}}} \sinh \left[\sqrt{\Omega_{k}} \int_{0}^{z_{2}} \frac{d z}{E(z)}\right],
$$

where $z_{2}$ is the value of redshift at the last scattering surface. From 7-year WMAP data [63], the value of the parameter has been obtained as $\mathscr{R}=1.726 \pm 0.018$ at the redshift $z_{2}=1091.3$. Now the $\chi^{2}$ function for the CMB measurement can be written as

$$
\chi_{\mathrm{CMB}}^{2}=\frac{(\mathscr{R}-1.726)^{2}}{(0.018)^{2}} .
$$




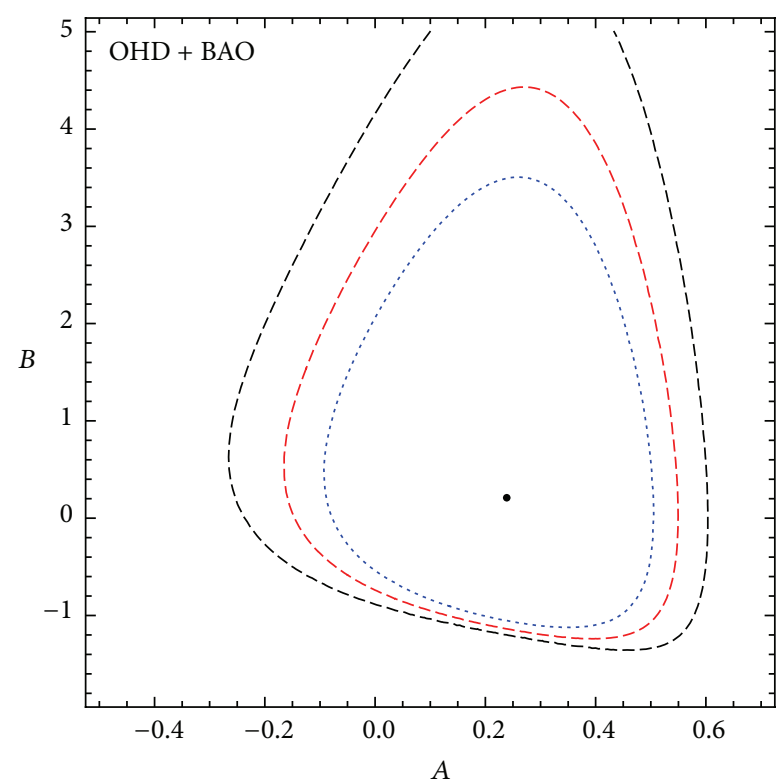

FIGURE 2: It shows the variations of $A$ with $B$ for $\alpha=0.1$ for OHD and OHD + BAO analysis, respectively, for different confidence levels say 66\% (solid, blue), 90\% (dashed, red), and 99\% (dashed, black) contours.

Now when we consider $\mathrm{OHD}, \mathrm{BAO}$, and $\mathrm{CMB}$ analysis together, the total joint data analysis $(\mathrm{OHD}+\mathrm{BAO}+\mathrm{CMB})$ for the $\chi^{2}$ function may be defined by

$$
\chi_{\mathrm{TOTAL}}^{2}=\chi_{\mathrm{OHD}}^{2}+\chi_{\mathrm{BAO}}^{2}+\chi_{\mathrm{CMB}}^{2} .
$$

Now the best fit values of $A$ and $B$ with $\chi^{2}$ for joint analysis of $\mathrm{BAO}$ and $\mathrm{CMB}$ with $\mathrm{OHD}$ observational data support the theoretical range of the parameters. The best fit values are $A=$ 0.239018 and $B=0.240047$ with the minimum value of $\chi^{2}=$ 7.07086. The $66 \%, 90 \%$, and $99 \%$ contours for $A$ and $B$ are plotted in Figure 3.

4.4. Redshift-Magnitude Observations from Supernovae Type Ia: Analysis with $\mathrm{OHD}+\mathrm{BAO}+\mathrm{CMB}+\mathrm{SNe} \mathrm{Ia}$. The observations of type Ia supernovae (SNe Ia) provide an excellent tool for probing the expansion history of the universe. The main evidence for the existence of dark energy is provided by the supernova type Ia experiments [1-3]. The type Ia observations directly measure the distance modulus of supernovae and its redshift $z[64,65]$. Now, take recent observational data (including SNe Ia) which consists of 557 data points and belongs to the Union2 sample [66]. From the type Ia observations, the luminosity distance determines the dark energy density. The luminosity distance $d_{L}(z)$ is defined by

$$
d_{L}(z)=\frac{(1+z)}{\sqrt{\Omega_{k}}} \sinh \left[\sqrt{\Omega_{k}} \int_{0}^{z} \frac{d z^{\prime}}{E\left(z^{\prime}\right)}\right]
$$

and the distance modulus $\mu(z)$ for supernovas is given by

$$
\mu(z)=5 \log _{10}\left[\frac{d_{L}(z) / H_{0}}{1 \mathrm{MPc}}\right]+25 .
$$

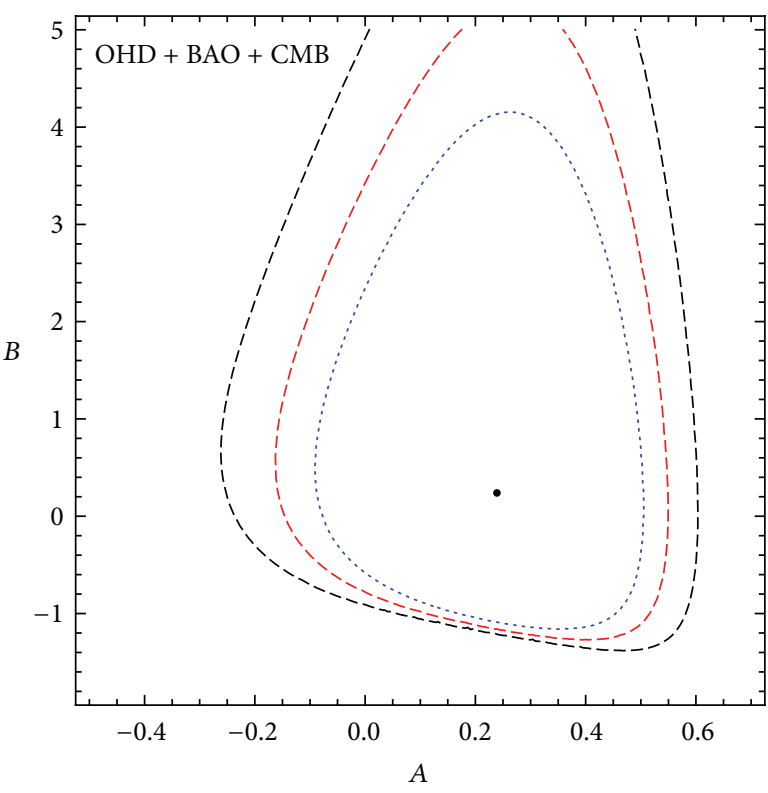

Figure 3: It shows the variations of $A$ with $B$ for $\alpha=0.1$ for OHD + $\mathrm{BAO}+\mathrm{CMB}$ and $\mathrm{OHD}+\mathrm{BAO}+\mathrm{CMB}+\mathrm{SNe}$ analysis, respectively, for different confidence levels say $66 \%$ (solid, blue), 90\% (dashed, red), and 99\% (dashed, black) contours.

The $\chi^{2}$ function for SNe Ia is given by

$$
\chi_{\mathrm{SNe}}^{2}=\sum \frac{\left(\mu(z)-\mu_{\mathrm{obs}}(z)\right)^{2}}{\sigma^{2}(z)},
$$

where $\mu_{\text {obs }}(z)$ is observational value of distance modulus parameter at different redshifts and $\sigma(z)$ is the corresponding error. In this work, we take Union2 data set consisting of 557 supernovae data points. Now we consider four cosmological tests together, the total joint data analysis (Stern $+\mathrm{BAO}+$ $\mathrm{CMB}+\mathrm{SNe}$ ) for the $\chi^{2}$ function may be defined by

$$
\chi_{\mathrm{TOTAL}}^{2}=\chi_{\mathrm{OHD}}^{2}+\chi_{\mathrm{BAO}}^{2}+\chi_{\mathrm{CMB}}^{2}+\chi_{\mathrm{SNe} e}^{2} .
$$

From the joint analysis, we found the minimum value of $\chi^{2}$ which is 7.06716. The best fit values of the parameters are $A=0.239158$ and $B=0.255814$. The confidence contours are drawn in Figure 4 . The best fit value of distance modulus $\mu(z)$ for our theoretical model and the supernova type Ia union2 sample are drawn in Figure 5 for our best fit values of $A$ and $B$. From the curves, we see that the theoretical MCG model in Einstein-Aether gravity is in agreement with the union2 sample data.

\section{Discussions and Concluding Remarks}

We have assumed FRW model of the universe in EinsteinAether gravity filled with dark matter and modified Chaplygin gas (MCG) type dark energy. Dark matter has the equation of state parameter $w_{m}$, which is small. We assumed the dark matter and dark energy separately conserved and hence we found the solutions in this gravity. Since $F(K)$ is a free function of $K$, so we have chosen quadratic form of 


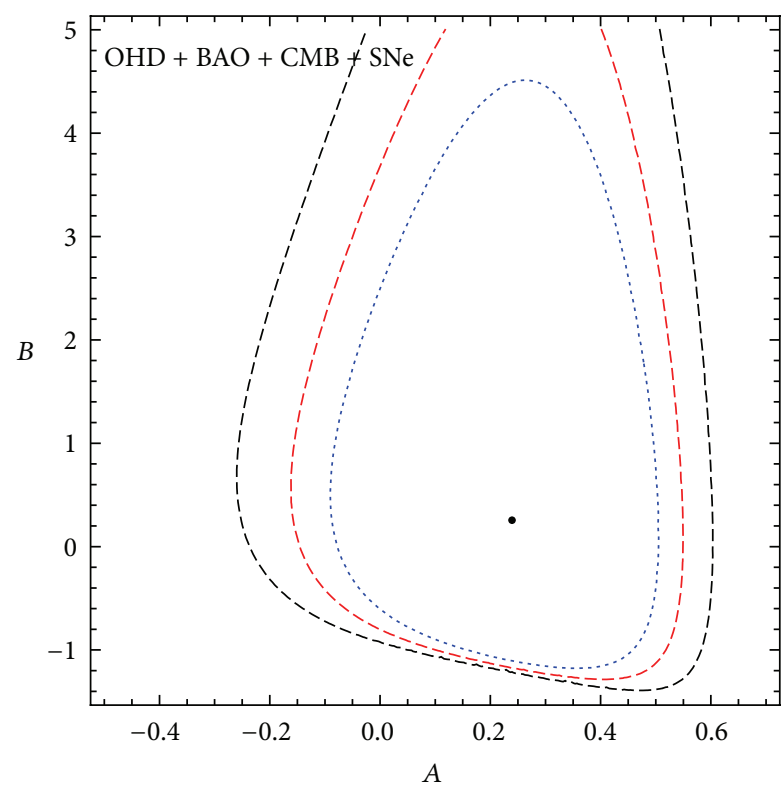

FIgURE 4: It shows the variations of $A$ with $B$ for $\alpha=0.1$ for OHD + $\mathrm{BAO}+\mathrm{CMB}$ and $\mathrm{OHD}+\mathrm{BAO}+\mathrm{CMB}+\mathrm{SNe}$ analysis, respectively, for different confidence levels say 66\% (solid, blue), 90\% (dashed, red), and 99\% (dashed, black) contours.

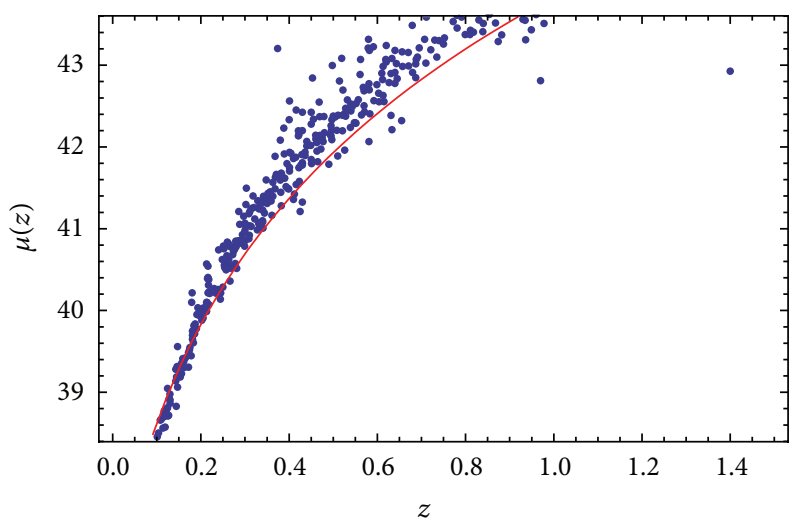

FIGURE 5: It shows the variation of distance modulus $\mu(z)$ versus redshift $z$ for our model (solid line) and the Union2 sample (dotted points).

$F(K)$ for simplicity of the calculation. Defining dimensionless parameters, we present the Hubble parameter in terms of some unknown parameters and observational parameters with the redshift $z$. From observed Hubble data (OHD) set (12 points), we have obtained the bounds of the arbitrary parameters $(A, B)$ of MCG by minimizing the $\chi^{2}$ test where we have chosen $\alpha=0.1$. The minimum values of the parameters are $A=0.238303$ and $B=0.18176$ for $\mathrm{OHD}$ analysis. Next due to joint analysis of $\mathrm{BAO}$ and $\mathrm{CMB}$ observations, we have also obtained the best fit values and the bounds of the parameters $(A, B)$. The best fit values of the parameters (i) for OHD + BAO are $A=0.238695$ and $B=$ 0.209932 and (ii) for OHD + BAO + CMB are $A=0.239018$ and $B=0.240047$. We have also taken type Ia supernovae data set (union 2 data set with 557 data points). Next due to joint analysis with $\mathrm{SNe}$, we have obtained the best fit values of the parameters $(A, B)$. The best fit values of the parameters for $\mathrm{OHD}+\mathrm{BAO}+\mathrm{CMB}+\mathrm{SNe}$ are $A=0.239158$ and $B=$ 0.255814 . The best fit values and bounds of the parameters are obtained by $66 \%, 90 \%$, and $99 \%$ confidence levels for OHD, $\mathrm{OHD}+\mathrm{BAO}, \mathrm{OHD}+\mathrm{BAO}+\mathrm{CMB}$, and $\mathrm{OHD}+\mathrm{BAO}+\mathrm{CMB}$ $+\mathrm{SNe}$ joint analysis in Figures 1-4. The distance modulus $\mu(z)$ against redshift $z$ for our theoretical MCG model in EinsteinAether gravity has been tested for the best fit values of the parameters and the observed SNe Ia union2 data sample and drawn in Figure 5. The observations do in fact severely constrain the nature of allowed composition of matter energy by constraining the range of the values of the parameters for a physically viable MCG in Einstein-Aether gravity model.

\section{Conflict of Interests}

The author declares that there is no conflict of interests regarding the publication of this paper.

\section{Acknowledgments}

The author is thankful to Institute of Theoretical Physics, Chinese Academy of Science, Beijing, China, for providing TWAS Associateship Programme under which part of the work was carried out. Also he is thankful to CSIR, Government of India, for providing research project Grant (no. 03(1206)/12/EMR-II).

\section{References}

[1] S. Perlmutter, G. Aldering, M. Della Valle et al., "Discovery of a supernova explosion at half the age of the Universe," Nature, vol. 391, no. 6662, pp. 51-54, 1998.

[2] A. G. Riess, A. V. Filippenko, P. Challis et al., "Observational evidence from supernovae for an accelerating universe and a cosmological constant," The Astronomical Journal, vol. 116, p. 1009, 1998.

[3] A. G. Riess, L.-G. Strolger, A. G. Riess et al., “Type la supernova discoveries at $z>1$ from the Hubble Space Telescope: evidence for past deceleration and constraints on dark energy evolution," The Astrophysical Journal, vol. 607, no. 2, p. 665, 2004.

[4] B. Boisseau, G. Esposito-Farèse, D. Polarski, and A. Starobinsky, "Reconstruction of a scalar-tensor theory of gravity in an accelerating universe," Physical Review Letters, vol. 85, no. 11, pp. 2236-2239, 2000.

[5] D. N. Spergel, R. Bean, O. Doré et al., “Three-year Wilkinson Microwave Anisotropy Probe (WMAP) observations: implications for cosmology," The Astrophysical Journal Supplement Series, vol. 170, no. 2, pp. 377-408, 2007.

[6] J. K. Adelman-McCarthy, M. A. Agüeros, S. S. Allam et al., "The sixth data release of the sloan digital sky survey," The Astrophysical Journal Supplement Series, vol. 175, no. 2, p. 297, 2008.

[7] D. J. Eisenstein, I. Zehavi, D. W. Hogg et al., "Detection of the Baryon acoustic peak in the large-scale correlation function of SDSS luminous red Galaxies," The Astrophysical Journal, vol. 633, no. 2, pp. 560-574, 2005. 
[8] S. L. Briddle, O. Lahav, J. P. Ostriker, and P. J. Steinhardt, "Precision cosmology? Not just yet," Science, vol. 299, pp. 15321533, 2003.

[9] D. N. Spergel, L. Verde, H. V. Peiris et al., "First-year Wilkinson Microwave Anisotropy Probe (WMAP) observations: determination of cosmological parameters," The Astrophysical Journal Supplement Series, vol. 148, no. 1, pp. 175-194, 2003.

[10] P. J. E. Peebles and B. Ratra, "Cosmology with a time-variable cosmological 'constant"' The Astrophysical Journal, vol. 325, pp. L17-L20, 1988.

[11] R. R. Caldwell, R. Dave, and P. J. Steinhardt, "Cosmological imprint of an energy component with general equation of state," Physical Review Letters, vol. 80, no. 8, pp. 1582-1585, 1998.

[12] C. Armendariz-Picon, V. Mukhanov, and P. J. Steinhardt, "Dynamical solution to the problem of a small cosmological constant and late-time cosmic acceleration," Physical Review Letters, vol. 85, no. 21, pp. 4438-4441, 2000.

[13] A. Sen, "Tachyon matter," Journal of High Energy Physics, vol. 2002, no. 7, p. 065, 2002.

[14] R. R. Caldwell, "A phantom menace? Cosmological consequences of a dark energy component with super-negative equation of state," Physics Letters B, vol. 545, no. 1-2, pp. 23-29, 2002.

[15] B. Feng, X. L. Wang, and X. M. Zhang, "Dark energy constraints from the cosmic age and supernova," Physics Letters B, vol. 607, no. 1-2, pp. 35-41, 2005.

[16] A. Kamenshchik, U. Moschella, and V. Pasquier, "An alternative to quintessence," Physics Letters B, vol. 511, no. 2-4, pp. 265-268, 2001.

[17] U. Debnath, A. Banerjee, and S. Chakraborty, "Role of modified Chaplygin gas in accelerated universe," Classical and Quantum Gravity, vol. 21, no. 23, pp. 5609-5617, 2004.

[18] A. G. Cohen, D. B. Kaplan, and A. E. Nelson, "Effective field theory, black holes, and the cosmological constant," Physical Review Letters, vol. 82, no. 25, pp. 4971-4974, 1999.

[19] V. Sahni and Y. Shtanov, "Brane world models of dark energy," Journal of Cosmology and Astroparticle Physics, vol. 2003, no. 11, p. 014, 2003.

[20] R.-G. Cai, "A dark energy model characterized by the age of the universe," Physics Letters B, vol. 657, no. 4-5, pp. 228-231, 2007.

[21] H. Wei and R. G. Cai, "A new model of agegraphic dark energy," Physics Letters B, vol. 660, no. 3, pp. 113-117, 2008.

[22] T. R. Choudhury and T. Padmanabhan, "Cosmological parameters from supernova observations: a critical comparison of three data sets," Astronomy \& Astrophysics, vol. 429, p. 807, 2007.

[23] J. L. Tonry, B. P. Schmidt, B. Barris et al., "Cosmological results from high-z supernovae," The Astrophysical Journal, vol. 594, no. 1, p. 1, 2003.

[24] B. J. Barris, J. L. Tonry, S. Blondin et al., "Twenty-three high-redshift supernovae from the Institute for Astronomy Deep survey: doubling the supernova sample at $z>0.7$," The Astrophysical Journal, vol. 602, no. 2, pp. 571-594, 2004.

[25] J. Lu, L. Xu, J. Li, B. Chang, Y. Gui, and H. Liu, "Constraints on modified Chaplygin gas from recent observations and a comparison of its status with other models," Physics Letters B, vol. 662, no. 2, pp. 87-91, 2008.

[26] L. Dao-Jun and L. Xin-Zhou, "Cosmic microwave background radiation constraints on a modified Chaplygin gas model," Chinese Physics Letters, vol. 22, no. 7, p. 1600, 2005.

[27] G. Dvali, G. Gabadadze, and M. Porrati, "Metastable gravitons and infinite volume extra dimensions," Physics Letters, Section B:
Nuclear, Elementary Particle and High-Energy Physics, vol. 484, no. 1-2, pp. 112-118, 2000.

[28] A. de Felice and T. Tsujikawa, “ $f(R)$ theories," Living Reviews in Relativity, vol. 13, article 3, 2010.

[29] S. Nojiri and S. D. Odintsov, "Unified cosmic history in modified gravity: from $\mathrm{F}(\mathrm{R})$ theory to Lorentz non-invariant models," Physics Reports, vol. 505, no. 2-4, pp. 59-144, 2011.

[30] T. Clifton and J. D. Barrow, "The power of general relativity," Physical Review, D: Third Series, vol. 72, no. 10, Article ID 103005, 2005.

[31] K. K. Yerzhanov, S. R. Myrzakul, I. I. Kulnazarov, and R. Myrzakulov, "Accelerating cosmology in $\mathrm{F}(\mathrm{T})$ gravity with scalar field," http://arxiv.org/abs/1006.3879.

[32] S. Nojiri and S. D. Odintsov, "Modified Gauss-Bonnet theory as gravitational alternative for dark energy," Physics Letters B, vol. 631, no. 1-2, pp. 1-6, 2005.

[33] I. Antoniadis, J. Rizos, and K. Tamvakis, "Singularity-free cosmological solutions of the superstring effective action," Nuclear Physics B, vol. 415, no. 2, pp. 497-514, 1994.

[34] P. Horava, "Membranes at quantum criticality," Journal of High Energy Physics, vol. 2009, no. 3, article 020, 2009.

[35] C. Brans and R. H. Dicke, "Mach's principle and a relativistic theory of gravitation," Physical Review, vol. 124, no. 3, pp. 925935, 1961.

[36] T. G. Zlosnik, P. G. Ferreira, and G. D. Starkman, "Modifying gravity with the aether: an alternative to dark matter," Physical Review D, vol. 75, no. 4, Article ID 044017, 2007.

[37] T. G. Zlosnik, P. G. Ferreira, and G. D. Starkman, "Growth of structure in theories with a dynamical preferred frame," Physical Review D: Particles, Fields, Gravitation and Cosmology, vol. 77, no. 8, Article ID 084010, 2008.

[38] T. Jacobson and D. Mattingly, "Gravity with a dynamical preferred frame," Physical Review D, vol. 64, no. 2, Article ID 024028, 9 pages, 2001.

[39] T. Jacobson and D. Mattingly, "Einstein-aether waves," Physical Review D, vol. 70, no. 2, Article ID 024003, 5 pages, 2004.

[40] D. Garfinkle and T. Jacobson, "A positive-energy theorem for Einstein-Aether and Hořava gravity," Physical Review Letters, vol. 107, Article ID 191102, 2011.

[41] E. V. Linder and R. J. Scherrer, "Aetherizing Lambda: barotropic fluids as dark energy," Physical Review D, vol. 80, no. 2, Article ID 023008, 7 pages, 2009.

[42] J. D. Barrow, "Some inflationary Einstein-Aether cosmologies," Physical Review D, vol. 85, Article ID 047503, 2012.

[43] J. Zuntz, T. G. Zlosnik, F. Bourliot, P. G. Ferreira, and G. D. Starkman, "Vector field models of modified gravity and the dark sector," Physical Review D, vol. 81, no. 10, Article ID 104015, 2010.

[44] B. Li, D. F. Mota, and J. D. Barrow, "Detecting a Lorentzviolating field in cosmology," Physical Review D: Particles, Fields, Gravitation, and Cosmology, vol. 77, no. 2, 2008.

[45] M. Gasperini, "Singularity prevention and broken Lorentz symmetry," Classical and Quantum Gravity, vol. 4, no. 2, pp. 485-494, 1987.

[46] M. Gasperini, "Repulsive gravity in the very early universe," General Relativity and Gravitation, vol. 30, no. 12, pp. 1703-1709, 1998.

[47] X. Meng and X. Du, "Einstein-aether theory as an alternative to dark energy model?" Physics Letters B, vol. 710, no. 4-5, pp. 493-499, 2012. 
[48] X. Meng and X. Du, "A specific case of generalized Einsteinaether theories," Communications in Theoretical Physics, vol. 57, no. 2, article 227, 2012.

[49] P. Wu and H. Yu, "Generalized Chaplygin gas model: constraints from Hubble parameter versus redshift data," Physics Letters B, vol. 644, no. 1, pp. 16-19, 2007.

[50] P. Thakur, S. Ghose, and B. C. Paul, "Modified Chaplygin gas and constraints on its $B$ parameter from cold dark matter and unified dark matter energy cosmological models," Monthly Notices of the Royal Astronomical Society, vol. 397, no. 4, pp. 1935-1939, 2009.

[51] B. C. Paul, P. Thakur, and S. Ghose, "Constraints on exotic matter for an emergent universe," http://arxiv.org/abs/1004.4256vl.

[52] B. C. Paul, S. Ghose, and P. Thakur, "Emergent Universe from a composition of matter, exotic matter and dark energy," Monthly Notices of the Royal Astronomical Society, vol. 413, no. 1, pp. 686690, 2011.

[53] S. Ghose, P. Thakur, and B. C. Paul, "Observational constraints on the model parameters of a class of emergent universe," Monthly Notices of the Royal Astronomical Society, vol. 421, no. 1, pp. 20-24, 2012.

[54] R. Biswas and U. Debnath, "Constraining redshift parametrization parameters of dark energy: loop quantum gravity as back ground," The European Physical Journal C, vol. 73, article 2424, 2013.

[55] S. Chakraborty, U. Debnath, and C. Ranjit, "Review of new physics effects in $t \bar{t}$ production," The European Physical Journal C, vol. 72, p. 2102, 2012.

[56] C. Ranjit, S. Chakraborty, and U. Debnath, "Observational constraints of homogeneous higher-dimensional cosmology with modified Chaplygin gas," The European Physical Journal Plus, vol. 128, article 53, 2013.

[57] C. Ranjit, S. Chakraborty, and U. Debnath, "Observational study of higher dimensional magnetic universe in non-linear electrodynamics," Astrophysics and Space Science, vol. 346, no. 1, pp. 291-299, 2013.

[58] D. Stern, R. Jimenez, L. Verde, M. Kamionkowski, and S. A. Stanford, "Cosmic chronometers: constraining the equation of State of dark energy. I: H(z) measurements," JCAP, vol. 1002, p. 008, 2010.

[59] T.-J. Zhang, C. Ma, and T. Lan, "Constraints on the dark side of the universe and observational Hubble parameter data," Advances in Astronomy, vol. 2010, Article ID 184284, 14 pages, 2010.

[60] J. R. Bond, G. Efstathiou, and M. Tegmark, "Forecasting cosmic parameter errors from microwave background anisotropy experiments," Monthly Notices of the Royal Astronomical Society, vol. 291, pp. L33-L41, 1997.

[61] G. Efstathiou and J. R. Bond, "Cosmic confusion: degeneracies among cosmological parameters derived from measurements of microwave background anisotropies," Monthly Notices of the Royal Astronomical Society, vol. 304, no. 1, pp. 75-97, 1999.

[62] S. Nessaeris and L. Perivolaropoulos, "Crossing the phantom divide: theoretical implications and observational status," Journal of Cosmology and Astroparticle Physics, vol. 2007, no. 01, p. 018, 2007.

[63] E. Komatsu, K. M. Smith, J. Dunkley et al., "Seven-year Wilkinson Microwave Anisotropy Probe (WMAP) observations: cosmological interpretation," The Astrophysical Journal Supplement Series, vol. 192, no. 2, p. 18, 2011.
[64] A. G. Riess, L.-G. Strolger, S. Casertano et al., "New hubble space telescope discoveries of type Ia supernovae at $z \geq 1$ : narrowing constraints on the early behavior of dark energy," The Astrophysical Journal, vol. 659, no. 1, pp. 98-121, 2007.

[65] M. Kowalaski, D. Rubin, G. Aldering et al., "Improved cosmological constraints from new, old, and combined supernova data sets," The Astrophysical Journal, vol. 686, no. 2, p. 749, 2008.

[66] R. Amanullah, C. Lidman, D. Rubin et al., "Spectra and hubble space telescope light curves of six type Ia supernovae at $0.511<$ $z<1.12$ and the union 2 compilation," The Astrophysical Journal, vol. 716, no. 1, p. 712, 2010. 

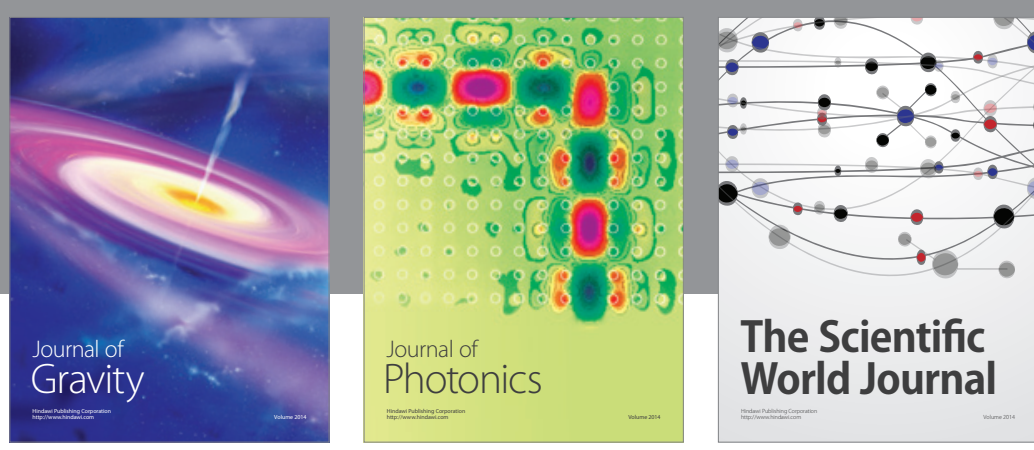

The Scientific World Journal
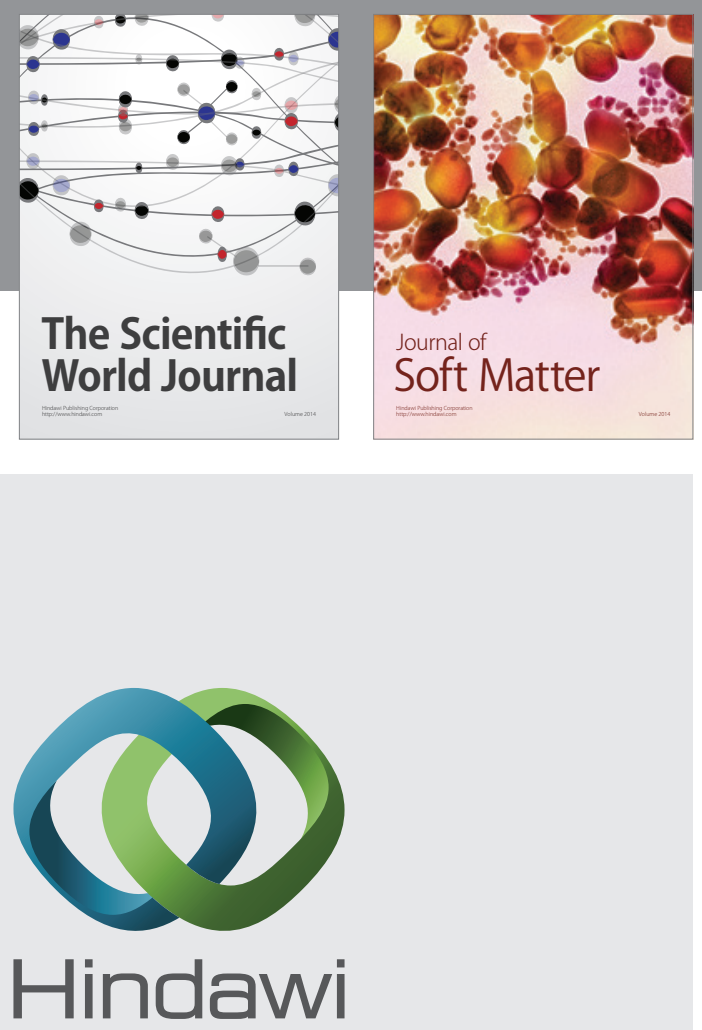

Submit your manuscripts at

http://www.hindawi.com

nternational Journal of

Statistical Mechanics
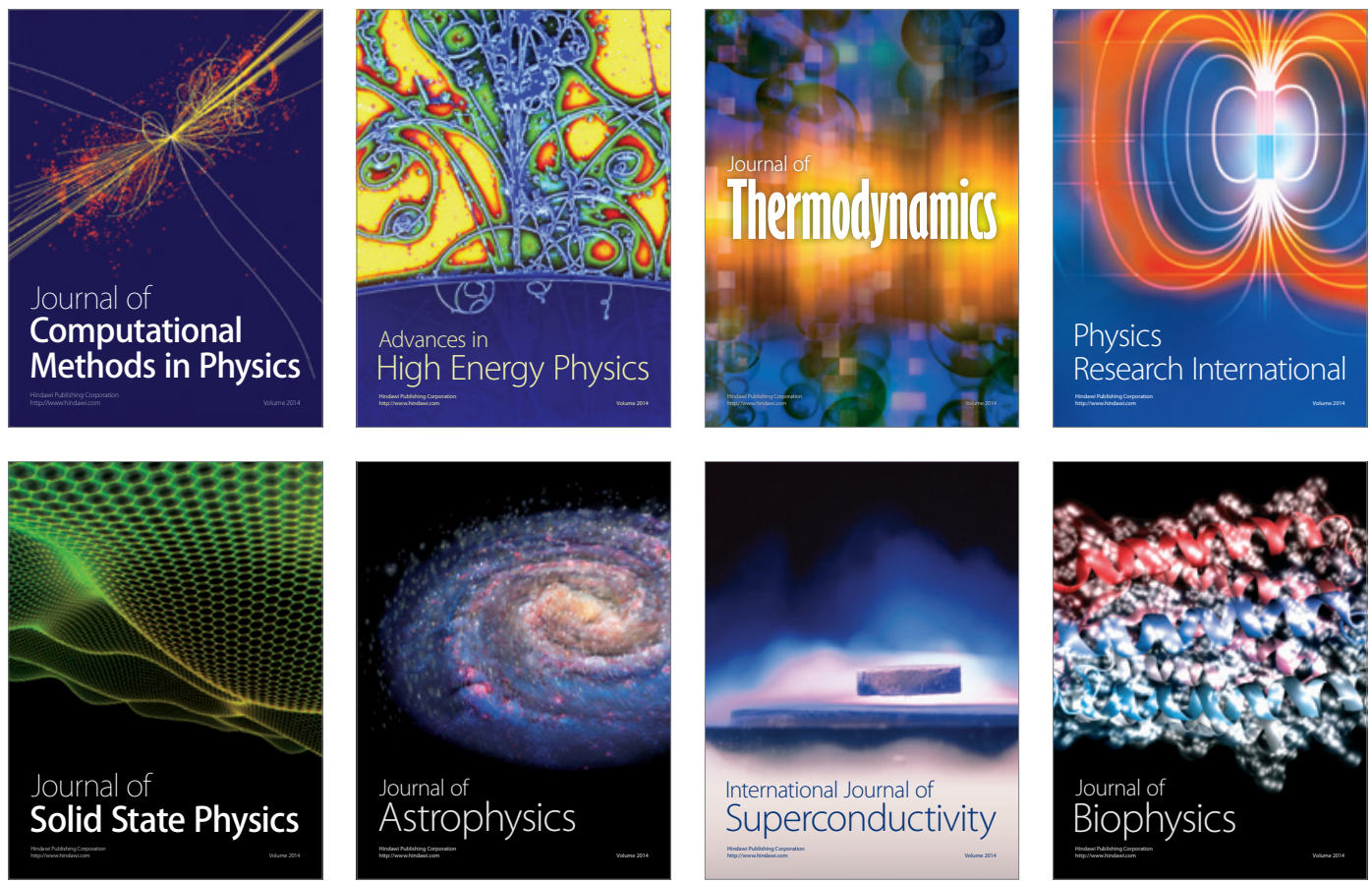
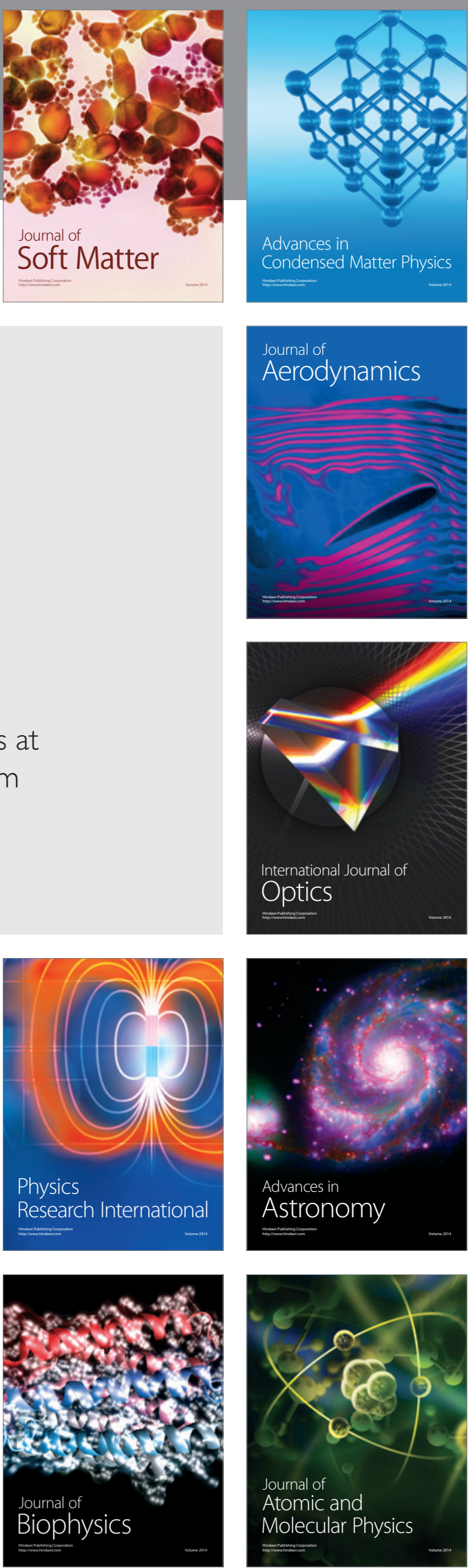